\title{
Pudendal nerve blocks in men undergoing urethroplasty: a case series
}

\author{
Arun Kalava, Abby M. Pribish, Lucas R. Wiegand
}

Department of Anesthesiology and Department of Urology, Tampa General Hospital, University of South Florida Morsani College of Medicine, USA

\begin{abstract}
The pudendal nerve block (PNB) is widely used for regional anesthesia during obstetric and anorectal procedures, but its role in urologic procedures has not been thoroughly studied. While transvaginal PNB is relatively straightforward, PNB in male patients often requires imaging guidance due to difficulty appreciating anatomic landmarks. We review the PNB and relevant sonoanatomy, and describe its analgesic efficacy in three male patients undergoing urethroplasty for urethral stricture. In this procedure, the patient was placed in lithotomy position, the ischial tuberosity was palpated, and the sacrotuberous ligament and pudendal artery were identified using ultrasound. Ropivacaine was injected medial to the pudendal artery and disappearance of muscle twitch was demonstrated. Two patients reported well-controlled pain at 24 hours postoperatively. One reported perineal pain requiring additional analgesia. All patients were discharged on postoperative day 1 without complications. Ultrasound-guided PNB provides safe and reasonably effective pain control to male patients undergoing urologic procedures.
\end{abstract}

Keywords: pudendal nerve, nerve block, ultrasound, regional anesthesia, male urologic surgery

Rom J Anaesth Intensive Care 2017; 24: 159-162

\section{Introduction}

The pudendal nerve block (PNB) has wide utility, including diagnosing pudendal neuralgia and providing perineal anesthesia during obstetric, anorectal and urologic procedures. PNBs are routinely performed in women via a transvaginal approach using well-defined anatomic landmarks, namely the ischial spines. This nerve block is more technically challenging in men, requiring an alternative approach such as transrectal, transperineal, transgluteal techniques, which many physicians are less comfortable and less experienced with performing. This procedure typically requires mul-

Address for correspondence:

Arun Kalava, MD

Tampa General Hospital

1 Tampa General Circle,

Tampa, FL 33606, USA

E-mail: arunkalava@yahoo.com tiple injections, a potentially increased amount of time depending on the procedura list, and special equipment including C-arm fluoroscopy, nerve stimulators, and ultrasonography [1]. This complexity often leads to the underuse of the PNB in men. While the merits of PNB in anorectal procedures such as hemorrhoidectomy [24] and transrectal ultrasound (TRUS)-guided prostate biopsy [5] are well studied, its utility in urologic procedures has not been as well described. We report a series of three male patients who received ultrasound and nerve stimulator guided PNB for postoperative analgesia following urethroplasty. We describe the PNB procedure and its relevant sonoanatomy.

\section{Case report}

Written informed consent was obtained from three patients who were scheduled for urethroplasty for treatment of urethral stricture disease. All three patients were male, ages 51,32, and 26. Two patients underwent anterior urethroplasty, and one patient underwent 
posterior urethroplasty. Under general endotracheal anesthesia, the patients were placed in dorsal lithotomy position, and the perineal area was prepared and draped in typical sterile fashion. A 21 gauge $9 \mathrm{~cm}$ Insulated Plexus (Arrow® Teleflex, Wayne, PA, USA) nerve stimulator needle was used for anesthetic infiltration bilaterally.

After palpating the ischial tuberosity, a high frequency (15-6 MHz) linear ultrasound probe was placed on the ischial tuberosity in a slanting vertical orientation (Fig. 1). The ultrasound transducer was then moved medially and the sacrotuberous ligament was identified. The internal pudendal artery was then identified under the sacrotuberous ligament using a color Doppler (Fig. 2). The stimuplex needle was then inserted in plane, medial to the pudendal artery stimulating at 1.5-2.0 $\mathrm{mA}$ and reducing the stimulus to $0.8-0.6 \mathrm{~mA}$ after eliciting perianal, scrotal and penile twitch. After negative aspiration of blood and successful twitch extenuation evidenced by Raj test, $15 \mathrm{~mL}$ of $0.5 \%$ ropivacaine was injected in $5 \mathrm{ml}$ increments. The needle insertion depth ranged from $4 \mathrm{~cm}$ to $5.5 \mathrm{~cm}$, depending on the patient's body habitus. The procedure was then repeated contralaterally. All patients tolerated the procedure well, and the duration of the procedure was 10-15 minutes. Strict aseptic technique was followed throughout the procedure.

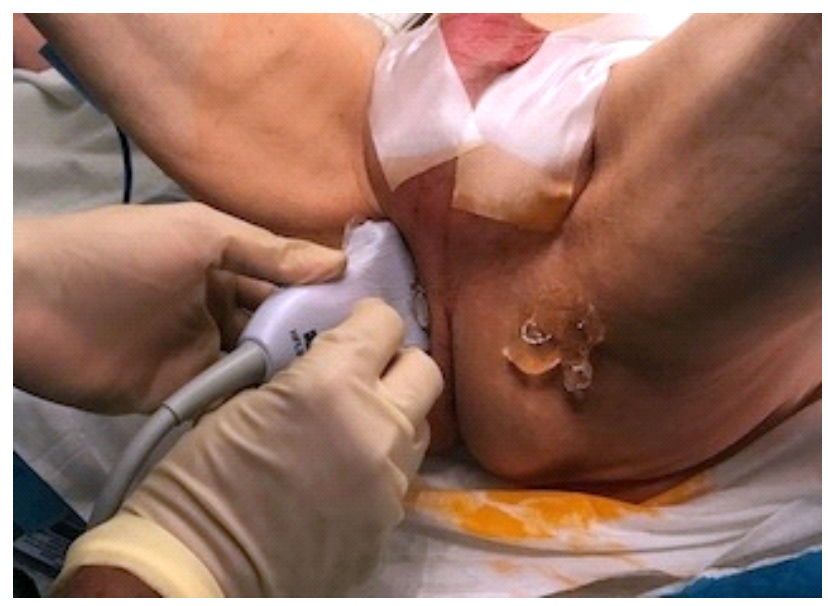

Fig. 1. High frequency ultrasound probe placement medial to the ischial tuberosity to visualize the sacrotuberous ligament and pudendal vessels

At 24 hours postoperatively, one patient denied perineal pain, one patient reported well-controlled perineal pain, and one patient reported moderate perineal pain requiring addition of topical ice packs to his analgesic regimen. Only one patient reported nausea postoperatively, which resolved with ondansetron. One patient reported numbness and paresthesias of the right foot which was suspected to represent compression

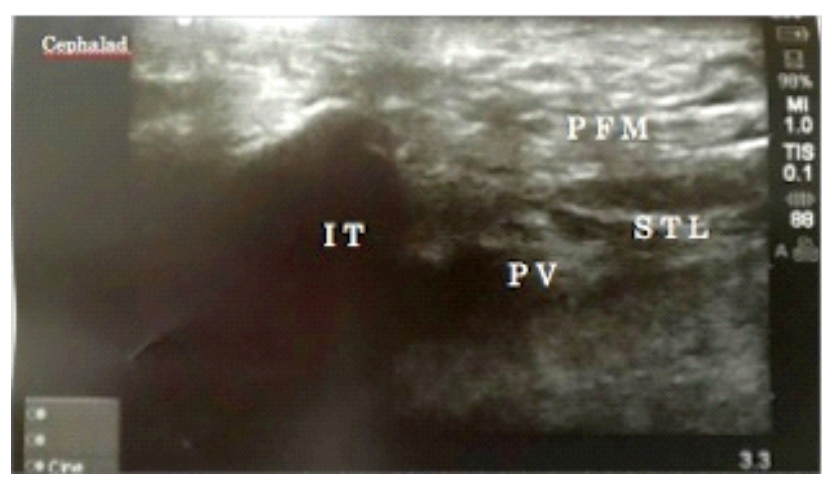

Fig. 2. Ultrasound image of pelvic anatomy: IT: Ischial Tuberosity; STL: Sacrotuberous Ligament; PFM: Pelvic Floor Muscles; PV: Pudendal Vessels

neuropathy from prolonged lithotomy, but this resolved prior to follow up at one month postoperatively. No other complications were reported. All patients were discharged on postoperative day (POD) 1 with oral pain medication. The effect of PNB on voiding could not be assessed, as two patients were discharged with a Foley catheter in place, and one patient was discharged with a suprapubic catheter to gravity drainage. At outpatient follow-up at 3-4 weeks postoperatively, all three patients were afebrile with no pain or minimal pain at the surgical site. One patient reported mild scrotal numbness. No other complications were reported.

\section{Discussion}

The PNB has wide applications, including providing perineal anesthesia during obstetric procedures including vaginal delivery, and anorectal procedures including hemorrhoidectomy and TRUS-guided prostate biopsy. Furthermore, pain relieved by PNB is an essential criterion for diagnosis of pudendal neuralgia by pudendal nerve entrapment syndrome [6]. The efficacy of PNB in male patients undergoing urologic procedures is not well established, although existing studies have shown promising results. For example, one study of patients undergoing transurethral resection of the prostate (TURP) showed that PNB correlates with reduced postoperative analgesic requirement and reduced postoperative discomfort related to catheter use, bladder irrigation, and instrumentation of the prostate [7]. To our knowledge, our case series is the first formal study of PNB efficacy in patients undergoing urethroplasty.

As in all surgical procedures, postoperative pain control following urethroplasty must balance the side effects and complication risk associated with anesthesia with the deleterious effects of postoperative pain. Insufficient postoperative pain control has been 
associated with greater opioid requirement, prolonged hospitalization, increased rates of hospital readmission, and higher rates of postoperative nausea and vomiting [3]. In our case series of three patients undergoing urethroplasty for urethral stricture, two patients reported adequate pain control at 24 hours postoperatively and one patient reported moderate pain requiring additional analgesia. One patient experienced postoperative nausea without vomiting. All patients were discharged on POD 1, and no patients were rehospitalized.

Anatomical considerations: The pudendal nerve arises from the sacral plexus with contributions from ventral rami of the second through fourth sacral nerves. The pudendal nerve exits through the greater sciatic foramina inferior to the piriformis muscle, crosses the posterior aspect of the sacrospinous ligament at the level of the ischial spine, and reenters the pelvis through the lesser sciatic foramina, then courses through Alcock's canal posteroinferiorly and divides into the inferior rectal nerve, perineal nerve, and the dorsal nerve of the clitoris or penis. The analgesic efficacy of the PNB during anorectal and urologic procedures arises from blocking the inferior rectal nerve, which supplies perianal skin, anoderm, and the external anal sphincter, and the perineal nerve, which has a superficial branch that supplies the labia or scrotum and a deep branch that supplies the external anal sphincter and urethral sphincter and mucosa [2,8]. Multiple approaches to the PNB have been described, including transvaginal, transperineal, transgluteal, and transrectal routes [1]. In female patients, the ischial spine is easily palpated transvaginally with the patient in lithotomy position, and anesthetic is typically injected posteriorly to the ischial spine at the attachment of the sacrospinous ligament, often without the use of image guidance [9]. The ischial spine is an important landmark for both nonguided and image-guided PNB. Injecting anesthetic medial to the ischial spine maximizes distance of the needle trajectory from important structures such as nerves and the pudendal artery [10]. Both the ischial spine and the sacrospinous ligament can be visualized easily using ultrasound. Of note, the pudendal nerve itself may not be visualized with ultrasound due to the variability in nerve size, and the pudendal nerve may consist of one to three trunks of varying size [11]. Many anesthesiologists may be less familiar with identifying ischial spines transrectally in men and perform this procedure infrequently, and thus the success rate for PNB is largely operator dependent [12].

Special equipment is typically required for PNB in male patients, such as ultrasonography, computerized tomography $(\mathrm{CT})$, or C-arm fluoroscopy. In the C-arm fluoroscopy technique described by Abdi et al. 2004, the $\mathrm{C}$-arm is used in the anteroposterior position at the level of the femoral heads to visualize the pelvic inlet of the prone patient, the ischial spine is visualized by angulating the fluoroscope obliquely, and anesthetic is injected at the tip of the ischial spine [9]. In a study of 53 patients with pudendal neuralgia by Antolak et al. 2016, fluoroscopically guided PNB led to complete pain relief at 2 hours in $39.2 \%$ of patients, and no anesthetic response in only $8.1 \%$ of patients [13]. While fluoroscopically-guided PNB is effective and reliable, like CT-guided PNB, drawbacks to this technique include higher cost and radiation exposure.

In contrast, ultrasound-guided PNB avoids radiation exposure to the patient and minimizes cost while providing the safety benefit of an image-guided procedure. Ultrasound guidance allows for easy identification of landmarks including the ischial spine and sacrotuberous ligament. Ultrasound guidance reduces the incidence of complications compared with nonguided PNB by allowing for easy identification of anatomic variants. For example, Gruber et al. 2001 determined that in a cohort of 58 cadavers, the position of pudendal nerves ranged from $17.2 \mathrm{~mm}$ medial to $8 \mathrm{~mm}$ lateral to the pudendal artery, and the pudendal artery ranged from $10.2 \mathrm{~mm}$ medial to $11.1 \mathrm{~mm}$ lateral to the tip of the ischial spine [11]. Ultrasound-guidance allows anesthetic to be more accurately infiltrated for maximum effect while minimizing the rate of possible complications, such as pudendal artery puncture.

A nerve stimulator is often used independently or in conjunction with image-guided techniques in order to increase the accuracy of needle placement and adequacy of the nerve block. Patient satisfaction with the PNB has also been shown to increase when a nerve stimulator is used [4]. The pudendal nerve contains both sensory and motor fibers and can be blocked using the nerve stimulator technique because its motor fibers mediate effector muscle contraction when electric current is applied [3]. We utilized a nerve stimulator in conjunction with ultrasound-guidance to optimize accuracy and adequacy of nerve block, with good results in two out of three patients in the case series.

Compared with other routes of anesthesia including general, spinal, caudal, and local anesthesia, PNB has been shown to achieve comparable or superior pain control. Nerve blocks have been shown to provide up to 12-18 hours of postoperative analgesia [14]. Naja et al. 2004 studied 90 patients undergoing hemorrhoidectomy and determined that patients who received PNB had significantly superior pain relief, faster return to daily activities, reduced need for analgesics, and higher satisfaction than patients who received general anesthesia alone or general anesthesia and placebo nerve block [4]. Similarly, Adsan et al. 2004 studied 51 patients undergoing TRUS-guided prostate biopsy and showed that patients who received unilateral PNB reported significantly less pain during prostate biopsy 
and probe manipulation [5]. In our case series, patients received general anesthesia for urethroplasty, with PNB performed prior to the procedure and in one patient at the end of the operation to reduce postoperative pain. This protocol achieved adequate postoperative pain control at 24 hours in two out of three patients, without serious side effects or complications.

Regional anesthesia techniques such as PNB also minimize the risk of anesthesia-associated complications, including urinary retention, which makes PNB especially useful for ambulatory surgery. For example, caudal and spinal anesthesia frequently cause urinary retention due to disruption of the micturition reflex. Kim et al. 2005 showed a significantly lower rate of postoperative urinary retention in hemorrhoidectomy patients who received PNB instead of spinal anesthesia (7.5\% compared with $69.6 \%$ ), as well as a smaller required volume of analgesic and significantly longer and more effective postoperative pain control in the PNB cohort [2]. Similarly, Imbelloni et al. 2007 demonstrated spontaneous micturition in 98 of 100 hemorrhoidectomy patients after PNB [3]. This low rate of urinary retention makes PNB a useful option for analgesia in outpatient anorectal and urologic procedures. We were unable to assess for urinary retention in our study, as our patients were discharged with either a suprapubic catheter or Foley catheter in place following urethroplasty.

Possible drawbacks associated with PNB include pain associated with local injection, time associated with the procedure, added cost associated with requiring the presence of an anesthesiologist [12], and risk associated with injecting anesthetic in a highly vascular area in close proximity to critical structures including the bladder and pudendal artery. Pain associated with local injection can be mitigated with preoperative treatment with an analgesic such as fentanyl $50 \mathrm{mcg}$ [2] or subcutaneous $2 \%$ lidocaine hydrochloride with adrenaline [15]. The risk of blindly injecting during PNB is mitigated by use of imageguided techniques such as ultrasonography. For each case in this series, PNB was performed in 10-15 minutes, which is similar to or shorter than the duration reported by other studies [2, 15]. Pain with local injection was avoided in our study, as patients were under general anesthesia at the time of this procedure.

Use of PNB correlates with high patient satisfaction and leads to cost savings for both the patient and the hospital by contributing to shorter hospitalizations and more expeditious return of the patient to daily activities, as well as avoiding cost associated with fluoroscopically or CT-guided procedures. Ultrasound-guided PNB can be used to provide effective, safe, and relatively cheap regional anesthesia in male patients undergoing anorectal or urological procedures, including urethroplasty.

\section{Conflict of interest}

Nothing to declare

\section{References}

1. Kim SH, Song SG, Paek OJ, Lee HJ, Park DH, Lee JK. Nervestimulator-guided pudendal nerve block by pararectal approach. Colorectal Dis 2012; 14: 611-615. DOI: 10.1111/j.14631318.2011.02720.x

2. Kim J, Lee DS, Jang SM, Shim MC, Jee DL. The effect of pudendal block on voiding after hemorrhoidectomy. Dis Colon Rectum 2005; 48: 518-523. DOI: 10.1007/s10350-004-0798-2

3. Imbelloni LE, Vieira EM, Gouveia MA, Netinho JG, Spirandelli LD, Cordeiro JA. Pudendal block with bupivacaine for postoperative pain relief. Dis Colon Rectum 2007; 50: 1656-1661. DOI: $10.1007 / \mathrm{s} 10350-007-0216-7$

4. Naja Z, Ziade MF, Lönnqvist PA. Nerve stimulator guided pudendal nerve block decreases posthemorrhoidectomy pain. Can J Anaesth 2005; 52: 62-68. DOI: 10.1007/BF03018582

5. Adsan O, Inal G, Ozdogan L, Kaygisiz O, Ugurlu O, Cetinkaya M. Unilateral pudendal nerve blockade for relief of all pain during transrectal ultrasound-guided biopsy of the prostate: a randomized, double-blind, placebo-controlled study. Urology 2004; 64: 528-531. DOI: 10.1016/j.urology.2004.04.033

6. Labat JJ, Riant T, Robert R, Amarenco G, Lefaucheur JP, Rigauld J. Diagnostic criteria for pudendal neuralgia by pudendal nerve entrapment (Nantes criteria). Neurourol Urodyn 2008; 27: 306310. DOI: 10.1002/nau.20505

7. Akkaya T, Ozkan D, Karakoyunlu N, Ergil J, Gumus H, Ersoy H, et al. Pudendal block in transurethral prostatectomy. Eur J Anaesthesiol 2015; 32: 656-657. DOI: 10.1097/EJA. 0000000000000172

8. Moore KL, Agur AMR, Dalley AF. Essential Clinical Anatomy. $4^{\text {th }}$ ed. Baltimore: Lippincott Williams \& Wilkins; 2011

9. Abdi S, Shenouda P, Patel N, Saini B, Bharat Y, Calvillo O. A novel technique for pudendal nerve block. Pain Physician 2004; 7: 319-322

10. Choi SS, Lee PB, Kim YC, Kim HJ, Lee SC. C-arm-guided pudendal nerve block: a new technique. Int J Clin Pract 2006; 60: 553-556. DOI: 10.1111/j.1742-1241.2006.00836.x

11. Gruber H, Kovacs P, Piegger J, Brenner E. New, simple, ultrasoundguided infiltration of the pudendal nerve: topographic basics. Dis Colon Rectum 2001; 44: 1376-1380

12. Maccagnano C, Scattoni V, Roscigno M, Raber M, Angiolilli D, Montorsi F, et al. Anaesthesia in transrectal prostate biopsy: which is the most effective technique? Urol Int 2011; 87: 1-13. DOI: $10.1159 / 000327827$

13. Antolak S Jr, Antolak C, Lendway L. Measuring the quality of pudendal nerve perineural injections. Pain Physician 2016; 19: 299-306

14. Avidan A, Drenger B, Ginosar Y. Peripheral nerve block for ambulatory surgery and postoperative analgesia. Curr Opin Anaesthesiol 2003; 16: 567-573

15. Mamlouk MD, vanSonnenberg E, Dehkharghani S. CT-Guided nerve block for pudendal neuralgia: diagnostic and therapeutic implications. AJR Am J Roentgenol 2014; 203: 196-200. DOI: 10.2214/AJR.13.11346 